\title{
Generalized hypergeometric distribution and its applications on univalent functions
}

\author{
Rajavadivelu Themangani ${ }^{1}$ (D), Saurabh Porwal ${ }^{2^{*}}$ (D) and Nanjundan Magesh ${ }^{3}$ (D)
}

"Correspondence:

saurabhjcb@rediffmail.com

${ }^{2}$ Department of Mathematics, Ram

Sahai Government Degree College,

Bairi-Shivrajpur, Kanpur 209205,

Uttar Pradesh, India

Full list of author information is

available at the end of the article

\begin{abstract}
The purpose of the present paper is to introduce a generalized hypergeometric distribution and obtain some necessary and sufficient conditions for generalized hypergeometric distribution series belonging to certain classes of univalent functions associated with the conic domains. We also investigate some inclusion relations. Finally, we discuss an integral operator related to this series.
\end{abstract}

MSC: $30 C 45$

Keywords: Analytic; Univalent functions; Uniformly convex and starlike functions; Generalized hypergeometric function; Probability distribution

\section{Introduction}

Let $\mathscr{A}$ denote the class of functions $f(z)$ of the form

$$
f(z)=z+\sum_{n=2}^{\infty} a_{n} z^{n}
$$

that are analytic in the open unit disk

$$
\mathbb{U}:=\{z \in \mathbb{C}:|z|<1\}
$$

and satisfy the normalized conditions $f(0)=f^{\prime}(0)-1=0$. As usual, we denote by $\mathscr{S}$ the subclass of $\mathscr{A}$ consisting of functions of the form (1.1) that are also univalent in $\mathbb{U}$. Further, by $\mathscr{T}$ we denote the subclass of $\mathscr{S}$ consisting of functions of the form

$$
f(z)=z-\sum_{n=2}^{\infty}\left|a_{n}\right| z^{n} .
$$

A function $f \in \mathscr{A}$ is said to be a starlike function of order $\alpha(0 \leq \alpha<1)$ if

$$
\Re\left(\frac{z f^{\prime}(z)}{f(z)}\right)>\alpha, \quad z \in \mathbb{U} .
$$

We denote the class of starlike functions of order $\alpha$ by $\mathscr{S}^{*}(\alpha)$.

(c) The Author(s) 2020. This article is licensed under a Creative Commons Attribution 4.0 International License, which permits use sharing, adaptation, distribution and reproduction in any medium or format, as long as you give appropriate credit to the original author(s) and the source, provide a link to the Creative Commons licence, and indicate if changes were made. The images or other third party material in this article are included in the article's Creative Commons licence, unless indicated otherwise in a credit line to the material. If material is not included in the article's Creative Commons licence and your intended use is not permitted by statutory regulation or exceeds the permitted use, you will need to obtain permission directly from the copyright holder. To view a copy of this licence, visit http://creativecommons.org/licenses/by/4.0/. 
A function $f \in \mathscr{A}$ is said to be a convex function of order $\alpha(0 \leq \alpha<1)$ if

$$
\Re\left(1+\frac{z f^{\prime \prime}(z)}{f^{\prime}(z)}\right)>\alpha, \quad z \in \mathbb{U} .
$$

We denote the class of convex functions of order $\alpha$ by $\mathscr{K}(\alpha)$.

Further, let $\mathscr{S}^{*}(0) \equiv \mathscr{S}^{*}$ and $\mathscr{K}(0) \equiv \mathscr{K}$ be the well-known standard classes of starlike and convex functions. The classes of starlike and convex functions of order $\alpha$ were studied earlier by Robertson [17] and Silverman [18].

For some $\alpha(0 \leq \alpha<1)$ and $\beta \geq 0$ and functions of the form (1.1), let $\mathscr{S}_{p}(\alpha, \beta)$ be the subclass of $\mathscr{S}$ satisfying the analytic criteria

$$
\Re\left(\frac{z f^{\prime}(z)}{f(z)}\right)-\alpha>\beta\left|\frac{z f^{\prime}(z)}{f(z)}-1\right|, \quad z \in \mathbb{U}
$$

and let $\mathscr{U} \mathscr{C} \mathscr{V}(\alpha, \beta)$ be the subclass of $\mathscr{S}$ satisfying the analytic criteria

$$
\Re\left(1+\frac{z f^{\prime \prime}(z)}{f^{\prime}(z)}\right)>\beta\left|\frac{z f^{\prime \prime}(z)}{f^{\prime}(z)}\right|+\alpha, \quad z \in \mathbb{U} .
$$

Note that $\mathscr{S}_{p}(\alpha, \beta) \cap \mathscr{T}=\mathscr{T} \mathscr{S}_{p}(\alpha, \beta)$ and $\mathscr{U} \mathscr{C} \mathscr{V}(\alpha, \beta) \cap \mathscr{T}=\mathscr{T} \mathscr{U} \mathscr{C} \mathscr{V}(\alpha, \beta)$.

The classes $\mathscr{S}_{p}(\alpha, \beta), \mathscr{U} \mathscr{C} \mathscr{V}(\alpha, \beta), \mathscr{T}_{p}(\alpha, \beta)$, and $\mathscr{T} \mathscr{U} \mathscr{C} \mathscr{V}(\alpha, \beta)$ were studied by Bharati et al. [3].

By specializing the parameters in $\mathscr{S}_{p}(\alpha, \beta)$ and $\mathscr{U} \mathscr{C} \mathscr{V}(\alpha, \beta)$ we obtain following known subclasses studied earlier by various researchers:

(1) $\mathscr{S}_{p}(0, \beta) \equiv \mathscr{S}_{p}(\beta)$ and $\mathscr{U} \mathscr{C} \mathscr{V}(0, \beta) \equiv \mathscr{U} \mathscr{C} \mathscr{V}(\beta)$ studied by Kanas and Wisniowska $[8,9]$.

(2) $\mathscr{S}_{p}(0,1) \equiv \mathscr{S}_{p}$ and $\mathscr{U} \mathscr{C} \mathscr{V}(0,1) \equiv \mathscr{U} \mathscr{C} \mathscr{V}$ studied by Goodman [6, 7] (see also [10]).

(3) $\mathscr{S}_{p}(\alpha, 0) \equiv \mathscr{S}^{*}(\alpha), \mathscr{U} \mathscr{C} \mathscr{V}(\alpha, 0) \equiv \mathscr{K}(\alpha), \mathscr{S}_{p}(0,0) \equiv \mathscr{S}^{*}$, and $\mathscr{U} \mathscr{C} \mathscr{V}(0,0) \equiv \mathscr{K}$ studied by Robertson [17] and Silverman [18].

In 1995, Dixit and Pal [4] introduced the class $\mathscr{R}^{\tau}(A, B)$ consisting of functions $f(z)$ of the form (1.1) that satisfy the inequality

$$
\left|\frac{f^{\prime}(z)-1}{(A-B) \tau-B\left(f^{\prime}(z)-1\right)}\right|<1, \quad \tau \in \mathbb{C} \backslash\{0\}, \quad-1 \leq B<A \leq 1, \quad z \in \mathbb{U} .
$$

For complex numbers $a_{1}, a_{2}, \ldots, a_{p}$ and $b_{1}, b_{2}, \ldots, b_{q}$ with $b_{j} \neq 0,-1,-2, \ldots, j=1,2, \ldots, q$, the generalized hypergeometric functions ${ }_{p} F_{q}\left(a_{1}, a_{2}, \ldots a_{p} ; b_{1}, b_{2}, \ldots b_{q} ; z\right)$ are defined by

$$
{ }_{p} F_{q}\left(a_{1}, a_{2}, \ldots a_{p} ; b_{1}, b_{2}, \ldots b_{q} ; z\right)=\sum_{n=0}^{\infty} \frac{\left(a_{1}\right)_{n} \cdots\left(a_{p}\right)_{n}}{\left(b_{1}\right)_{n} \cdots\left(b_{q}\right)_{n}} \frac{z^{n}}{n !}, \quad z \in \mathbb{U}
$$

where $p \leq q+1$, and $(a)_{n}$ is the Pochhammer symbol defined by

$$
(a)_{n}=\frac{\Gamma(a+n)}{\Gamma(a)}= \begin{cases}1 & \text { if } n=0 \\ a(a+1)(a+2) \ldots(a+n-1) & \text { if } n \in \mathbb{N}\end{cases}
$$

The convergence conditions for the series defined by (1.5) are as follows: 
(1) If $p<q+1$, then the series converges absolutely in the entire complex plane.

(2) If $p \leq q$, then the series converges absolutely for every finite $z$.

(3) If $p=q+1$, then the series converges absolutely for $|z|<1$.

(4) If $p=q+1$ and $|z|=1$, then the series converges when $\Re\left\{\sum_{j=1}^{q} b_{j}-\sum_{i=1}^{p} a_{i}\right\}>0$.

For a detailed study, we refer to [16].

Now for $a_{i}, i=1,2, \ldots, p, b_{j}, j=1,2, \ldots, q$, and $m>0$, we define

$$
{ }_{p} F_{q}\left(a_{1}, a_{2}, \ldots a_{p} ; b_{1}, b_{2}, \ldots b_{q} ; m\right)=\sum_{n=0}^{\infty} \frac{\left(a_{1}\right)_{n} \cdots\left(a_{p}\right)_{n}}{\left(b_{1}\right)_{n} \cdots\left(b_{q}\right)_{n}} \frac{m^{n}}{n !}
$$

provided that the series is convergent.

In this paper, we use the notations

$$
{ }_{p} F_{q}(z)={ }_{p} F_{q}\left(a_{1}, a_{2}, \ldots a_{p} ; b_{1}, b_{2}, \ldots b_{q} ; z\right)
$$

and

$$
{ }_{p} F_{q}\left(a_{1}+k ; b_{1}+k ; z\right)={ }_{p} F_{q}\left(a_{1}+k, a_{2}+k, \ldots a_{p}+k ; b_{1}+k, b_{2}+k, \ldots b_{q}+k ; z\right), \quad k \in \mathbb{N} \text {. }
$$

Now we introduce the generalized hypergeometric distribution with probability mass function

$$
\frac{\left(a_{1}\right)_{n} \cdots\left(a_{p}\right)_{n}}{\left(b_{1}\right)_{n} \cdots\left(b_{q}\right)_{n}} \frac{m^{n}}{n !} \frac{1}{{ }_{p} F_{q}(m)}, \quad n=0,1,2, \ldots
$$

By specializing the parameters in the generalized hypergeometric distribution it reduces to the following probability distributions:

(1) If $p=2$ and $q=1$, then it reduces to the hypergeometric-type probability distribution studied by Porwal and Gupta [15].

(2) If $p=q=1$, then it reduces to the confluent hypergeometric distribution studied by Porwal [14].

(3) If $p=q=1$ and $a_{1}=b_{1}$, then it reduces to the well-known Poisson distribution.

Next, we introduce the generalized hypergeometric distribution series whose coefficients are the probabilities of generalized hypergeometric distribution

$$
{ }_{p} F_{q}(m, z)=z+\sum_{n=2}^{\infty} \frac{\left(a_{1}\right)_{n-1} \cdots\left(a_{p}\right)_{n-1}}{\left(b_{1}\right)_{n-1} \cdots\left(b_{q}\right)_{n-1}} \frac{m^{n-1}}{(n-1) !} \frac{z^{n}}{{ }_{p} F_{q}(m)}
$$

where $a_{i}, b_{j}>0, i=1,2, \ldots, p, j=1,2, \ldots, q$.

Now we define

$$
{ }_{p} \bar{F}_{q}(m, z)=2 z-{ }_{p} F_{q}(m, z)=z-\sum_{n=2}^{\infty} \frac{\left(a_{1}\right)_{n-1} \cdots\left(a_{p}\right)_{n-1}}{\left(b_{1}\right)_{n-1} \cdots\left(b_{q}\right)_{n-1}} \frac{m^{n-1}}{(n-1) !} \frac{z^{n}}{{ }_{p} F_{q}(m)}
$$

The convolution (or Hadamard product) of two power series is defined as

$$
(f * g)(z)=f(z) * g(z)=\sum_{n=0}^{\infty} a_{n} b_{n} z^{n}, \quad z \in \mathbb{U}
$$


Now we consider the linear operator $\Omega(p, q, m): \mathscr{A} \rightarrow \mathscr{A}$ defined by

$$
\Omega(p, q ; m) f(z)={ }_{p} F_{q}(m, z) * f(z)=z+\sum_{n=2}^{\infty} \frac{\left(a_{1}\right)_{n-1} \cdots\left(a_{p}\right)_{n-1}}{\left(b_{1}\right)_{n-1} \cdots\left(b_{q}\right)_{n-1}} \frac{m^{n-1}}{(n-1) !} \frac{a_{n} z^{n}}{F_{q}(m)} .
$$

In 2014, Porwal [12] introduced the Poisson distribution series and obtained necessary and sufficient conditions for this series to belong to certain classes of univalent functions. It opens up a new and interesting direction of research in geometric function theory. After the appearance of this paper, several researchers introduced the hypergeometric distribution series [1], the binomial distribution series [11], the hypergeometric-type distribution series [15], the confluent hypergeometric distribution series [14], the Pascal distribution series [5], the generalized distribution series [13], and the Mittag-Leffler-type Poisson distribution series [2] and obtained some necessary and sufficient conditions for them to belong to certain classes of univalent functions. Motivated with the works mentioned, in this paper, we obtain some necessary and sufficient conditions for the generalized hypergeometric distribution series to belong to the classes $\mathscr{S}_{p}(\alpha, \beta), \mathscr{U} \mathscr{C} \mathscr{V}(\alpha, \beta)$, and $\mathscr{U} \mathscr{C} \mathscr{V}(\beta)$. We also obtain some inclusion relations between the classes $\mathscr{R}^{\tau}(A, B)$ and $\mathscr{U} \mathscr{C} \mathscr{V}(\alpha, \beta)$, $\mathscr{U} \mathscr{C} \mathscr{V}(\beta)$. Finally, we discuss an integral operator associated with the generalized distribution series.

\section{Main results}

To prove our main results, we need the following lemmas.

Lemma 2.1 ([13]) A function $f \in \mathscr{A}$ of the form (1.1) belongs to the class $\mathscr{S}_{p}(\alpha, \beta)$ if

$$
\sum_{n=2}^{\infty}[n(1+\beta)-(\alpha+\beta)]\left|a_{n}\right| \leq 1-\alpha .
$$

Lemma 2.2 ([3]) A function $f \in \mathscr{A}$ of the form (1.1) is said to be in the class $\mathscr{U} \mathscr{C} \mathscr{V}(\alpha, \beta)$ if

$$
\sum_{n=2}^{\infty} n[n(1+\beta)-(\alpha+\beta)]\left|a_{n}\right| \leq 1-\alpha .
$$

Remark 2.1 Conditions (2.1) and (2.2) are also necessary for functions $f(z)$ of the form (1.2).

Lemma 2.3 ([8]) A function $f \in \mathscr{A}$ of the form (1.1) is said to be in the class $\mathscr{U} \mathscr{C} \mathscr{V}(\beta)$ if

$$
\sum_{n=2}^{\infty} n(n-1)\left|a_{n}\right| \leq \frac{1}{\beta+2}
$$

The number $\frac{1}{\beta+2}$ cannot be increased.

Lemma 2.4 ([4]) Iff $\in \mathscr{R}^{\tau}(A, B)$ is of the form (1.1), then

$$
\left|a_{n}\right| \leq \frac{(A-B)|\tau|}{n}, \quad n \in \mathbb{N} \backslash\{1\} .
$$

The bounds given in (2.4) are sharp. 
Theorem 2.1 Let $a_{i}, b_{j}>0(i=1,2, \ldots, p ; j=1,2, \ldots, q)$. Suppose that the inequality

$$
\begin{aligned}
(1+\beta) & \frac{a_{1}\left(a_{1}+1\right) \cdots a_{p}\left(a_{p}+1\right)}{b_{1}\left(b_{1}+1\right) \cdots b_{q}\left(b_{q}+1\right)} m_{p}^{2} F_{q}\left(a_{1}+2 ; b_{1}+2 ; m\right) \\
& +(3+2 \beta-\alpha) \frac{a_{1} \cdots a_{p}}{b_{1} \cdots b_{q}} m_{p} F_{q}\left(a_{1}+1 ; b_{1}+1 ; m\right) \leq 1-\alpha
\end{aligned}
$$

holds with one of the following conditions:

(1) $p \leq q$ and $m>0$,

(2) $p=q+1$ and $m<1$,

(3) $p=q+1, m=1$, and $\sum_{j=1}^{q} b_{j}>\sum_{i=1}^{p} a_{i}+2$.

Then ${ }_{p} F_{q}(m, z)$ defined by (1.7) is in the class $\mathscr{U} \mathscr{C} \mathscr{V}(\alpha, \beta)$.

Proof To prove that ${ }_{p} F_{q}(m, z)$ defined by (1.7) is in the class $\mathscr{U} \mathscr{C} \mathscr{V}(\alpha, \beta)$, by Lemma 2.2 it suffices to prove that

$$
\sum_{n=2}^{\infty} n[n(1+\beta)-(\alpha+\beta)] \frac{\left(a_{1}\right)_{n-1} \cdots\left(a_{p}\right)_{n-1}}{\left(b_{1}\right)_{n-1} \cdots\left(b_{q}\right)_{n-1}} \frac{m^{n-1}}{(n-1) !} \frac{1}{{ }_{p} F_{q}(m)} \leq 1-\alpha
$$

We have

$$
\begin{aligned}
\sum_{n=2}^{\infty} n & {[n(1+\beta)-(\alpha+\beta)] \frac{\left(a_{1}\right)_{n-1} \cdots\left(a_{p}\right)_{n-1}}{\left(b_{1}\right)_{n-1} \cdots\left(b_{q}\right)_{n-1}} \frac{m^{n-1}}{(n-1) !} \frac{1}{{ }_{p} F_{q}(m)} } \\
= & \frac{1}{{ }_{p} F_{q}(m)}\left[\sum_{n=2}^{\infty}[(n-1)(n-2)(1+\beta)+(3+2 \beta-\alpha)(n-1)\right. \\
& \left.+(1-\alpha)] \frac{\left(a_{1}\right)_{n-1} \cdots\left(a_{p}\right)_{n-1}}{\left(b_{1}\right)_{n-1} \cdots\left(b_{q}\right)_{n-1}} \frac{m^{n-1}}{(n-1) !}\right] \\
= & \frac{1}{{ }_{p} F_{q}(m)}\left[(1+\beta) \sum_{n=2}^{\infty} \frac{\left(a_{1}\right)_{n-1} \cdots\left(a_{p}\right)_{n-1}}{\left(b_{1}\right)_{n-1} \cdots\left(b_{q}\right)_{n-1}} \frac{m^{n-1}}{(n-3) !}\right. \\
& +(3+2 \beta-\alpha) \sum_{n=2}^{\infty} \frac{\left(a_{1}\right)_{n-1} \cdots\left(a_{p}\right)_{n-1}}{\left(b_{1}\right)_{n-1} \cdots\left(b_{q}\right)_{n-1}} \frac{m^{n-1}}{(n-2) !} \\
& \left.+(1-\alpha) \sum_{n=2}^{\infty} \frac{\left(a_{1}\right)_{n-1} \cdots\left(a_{p}\right)_{n-1}}{\left(b_{1}\right)_{n-1} \cdots\left(b_{q}\right)_{n-1}} \frac{m^{n-1}}{(n-1) !}\right] \\
& \left.+(1-\alpha) \sum_{n=2}^{\infty} \frac{\left(a_{1}\right)_{n-1} \cdots\left(a_{p}\right)_{n-1}}{\left(b_{1}\right)_{n-1} \cdots\left(b_{q}\right)_{n-1}} \frac{m^{n-1}}{(n-1) !}\right] \\
& +(1+\beta) \frac{a_{1}\left(a_{1}+1\right) \cdots a_{p}\left(a_{p}+1\right)}{b_{1}\left(b_{1}+1\right) \cdots b_{q}\left(b_{q}+1\right)} m^{2}{ }_{p} F_{q}\left(a_{1}+2 ; b_{1}+2 ; m\right) \\
& +(1+\beta) \frac{a_{1}\left(a_{1}+1\right) \cdots a_{p}\left(a_{p}+1\right)}{b_{1}\left(b_{1}+1\right) \cdots b_{q}\left(b_{q}+1\right)} m^{2} \sum_{n=2}^{\infty} \frac{\left(a_{1}+2\right)_{n-3} \cdots\left(a_{p}+2\right)_{n-3}}{\left(b_{1}+2\right)_{n-3} \cdots\left(b_{q}+2\right)_{n-3}} \frac{m^{n-3}}{(n-3) !} \\
b_{1} \cdots b_{q} m_{n=2} & \frac{\left.a_{1} \cdots a_{p}+1\right)_{n-2} \cdots\left(a_{p}+1\right)_{n-2}}{(n-1)} \frac{m^{n-2}}{(n-2) !}
\end{aligned}
$$




$$
\begin{aligned}
& \left.+(3+2 \beta-\alpha) \frac{a_{1} \cdots a_{p}}{b_{1} \cdots b_{q}} m_{p} F_{q}\left(a_{1}+1 ; b_{1}+1 ; m\right)+(1-\alpha)\left({ }_{p} F_{q}(m)-1\right)\right] \\
\leq & 1-\alpha
\end{aligned}
$$

by the given hypothesis. This completes the proof of Theorem 2.1 .

Theorem 2.2 Let $a_{i}, b_{j}>0(i=1,2, \ldots, p ; j=1,2, \ldots, q)$. Suppose that the inequality

$$
(1+\beta) \frac{a_{1} \cdots a_{p}}{b_{1} \cdots b_{q}} m_{p} F_{q}\left(a_{1}+1 ; b_{1}+1 ; m\right) \leq 1-\alpha
$$

holds with one of the following conditions:

(1) $p \leq q$ and $m>0$,

(2) $p=q+1$ and $m<1$,

(3) $p=q+1, m=1$, and $\sum_{j=1}^{q} b_{j}>\sum_{i=1}^{p} a_{i}+1$.

Then ${ }_{p} F_{q}(m, z)$ defined by (1.7) is in the class $\mathscr{S}_{p}(\alpha, \beta)$.

Proof To prove that ${ }_{p} F_{q}(m, z)$ defined by $(1.7)$ is in the class $\mathscr{S}_{p}(\alpha, \beta)$, by Lemma 2.1 it suffices to prove that

$$
\sum_{n=2}^{\infty}[n(1+\beta)-(\alpha+\beta)] \frac{\left(a_{1}\right)_{n-1} \cdots\left(a_{p}\right)_{n-1}}{\left(b_{1}\right)_{n-1} \cdots\left(b_{q}\right)_{n-1}} \frac{m^{n-1}}{(n-1) !} \frac{1}{F_{q}(m)} \leq 1-\alpha .
$$

We have

$$
\begin{aligned}
\sum_{n=2}^{\infty}[ & n(1+\beta)-(\alpha+\beta)] \frac{\left(a_{1}\right)_{n-1} \cdots\left(a_{p}\right)_{n-1}}{\left(b_{1}\right)_{n-1} \cdots\left(b_{q}\right)_{n-1}} \frac{m^{n-1}}{(n-1) !} \frac{1}{{ }_{p} F_{q}(m)} \\
= & \frac{1}{{ }_{p} F_{q}(m)}\left[(1+\beta) \sum_{n=2}^{\infty}(n-1) \frac{\left(a_{1}\right)_{n-1} \cdots\left(a_{p}\right)_{n-1}}{\left(b_{1}\right)_{n-1} \cdots\left(b_{q}\right)_{n-1}} \frac{m^{n-1}}{(n-1) !}\right. \\
& \left.+(1-\alpha) \sum_{n=2}^{\infty} \frac{\left(a_{1}\right)_{n-1} \cdots\left(a_{p}\right)_{n-1}}{\left(b_{1}\right)_{n-1} \cdots\left(b_{q}\right)_{n-1}} \frac{m^{n-1}}{(n-1) !}\right] \\
= & \frac{1}{{ }_{p} F_{q}(m)}\left[(1+\beta) \frac{a_{1} \cdots a_{p}}{b_{1} \cdots b_{q}} m \sum_{n=2}^{\infty} \frac{\left(a_{1}+1\right)_{n-2} \cdots\left(a_{p}+1\right)_{n-2}}{\left(b_{1}+1\right)_{n-2} \cdots\left(b_{q}+1\right)_{n-2}} \frac{m^{n-2}}{(n-2) !}\right. \\
& \left.+(1-\alpha) \sum_{n=2}^{\infty} \frac{\left(a_{1}\right)_{n-1} \cdots\left(a_{p}\right)_{n-1}}{\left(b_{1}\right)_{n-1} \cdots\left(b_{q}\right)_{n-1}} \frac{m^{n-1}}{(n-1) !}\right] \\
= & \frac{1}{{ }_{p} F_{q}(m)}\left[(1+\beta) \frac{a_{1} \cdots a_{p}}{b_{1} \cdots b_{q}} m_{p} F_{q}\left(a_{1}+1 ; b_{1}+1 ; m\right)+(1-\alpha)\left({ }_{p} F_{q}(m)-1\right)\right] \\
\leq & 1-\alpha,
\end{aligned}
$$

by the given hypothesis. This completes the proof of Theorem 2.2 .

Remark 2.2 Conditions (2.5) and (2.6) are also necessary for the series ${ }_{p} \bar{F}_{q}(m, z)$ defined by (1.8) to belong to the classes $\mathscr{T} \mathscr{S}_{p}(\alpha, \beta)$ and $\mathscr{T} \mathscr{U} \mathscr{C} \mathscr{V}(\alpha, \beta)$, respectively. 
Theorem 2.3 Let $a_{i}, b_{j}>0(i=1,2, \ldots, p ; j=1,2, \ldots, q)$. Suppose that the inequality

$$
\begin{aligned}
& \frac{a_{1}\left(a_{1}+1\right) \cdots a_{p}\left(a_{p}+1\right)}{b_{1}\left(b_{1}+1\right) \cdots b_{q}\left(b_{q}+1\right)} m_{p}^{2} F_{q}\left(a_{1}+2 ; b_{1}+2 ; m\right)+2 \frac{a_{1} \cdots a_{p}}{b_{1} \cdots b_{q}} m_{p} F_{q}\left(a_{1}+1 ; b_{1}+1 ; m\right) \\
& \quad \leq \frac{{ }_{p} F_{q}(m)}{\beta+2}
\end{aligned}
$$

holds with one of the following conditions:

(1) $p \leq q$ and $m>0$,

(2) $p=q+1$ and $m<1$,

(3) $p=q+1, m=1$, and $\sum_{j=1}^{q} b_{j}>\sum_{i=1}^{p} a_{i}+2$.

Then ${ }_{p} F_{q}(m, z)$ defined by (1.7) is in the class $\mathscr{U} \mathscr{C} \mathscr{V}(\beta)$.

Proof To prove that ${ }_{p} F_{q}(m, z)$ defined by $(1.7)$ is in the class $\mathscr{U} \mathscr{C} \mathscr{V}(\beta)$, by Lemma 2.3 it suffices to prove that

$$
\sum_{n=2}^{\infty} n[n-1] \frac{\left(a_{1}\right)_{n-1} \cdots\left(a_{p}\right)_{n-1}}{\left(b_{1}\right)_{n-1} \cdots\left(b_{q}\right)_{n-1}} \frac{m^{n-1}}{(n-1) !} \frac{1}{{ }_{p} F_{q}(m)} \leq \frac{1}{\beta+2}
$$

We have

$$
\begin{aligned}
\sum_{n=2}^{\infty} n[n-1] & \frac{\left(a_{1}\right)_{n-1} \cdots\left(a_{p}\right)_{n-1}}{\left(b_{1}\right)_{n-1} \cdots\left(b_{q}\right)_{n-1}} \frac{m^{n-1}}{(n-1) !} \frac{1}{{ }_{p} F_{q}(m)} \\
= & \frac{1}{{ }_{p} F_{q}(m)}\left[\sum_{n=2}^{\infty} \frac{\left(a_{1}\right)_{n-1} \cdots\left(a_{p}\right)_{n-1}}{\left(b_{1}\right)_{n-1} \cdots\left(b_{q}\right)_{n-1}} \frac{m^{n-1}}{(n-3) !}+2 \sum_{n=2}^{\infty} \frac{\left(a_{1}\right)_{n-1} \cdots\left(a_{p}\right)_{n-1}}{\left(b_{1}\right)_{n-1} \cdots\left(b_{q}\right)_{n-1}} \frac{m^{n-1}}{(n-2) !}\right] \\
= & \frac{1}{{ }_{p} F_{q}(m)}\left[\frac{a_{1}\left(a_{1}+1\right) \cdots a_{p}\left(a_{p}+1\right)}{b_{1}\left(b_{1}+1\right) \cdots b_{q}\left(b_{q}+1\right)} m^{2} \sum_{n=2}^{\infty} \frac{\left(a_{1}+2\right)_{n-3} \cdots\left(a_{p}+2\right)_{n-3}}{\left(b_{1}+2\right)_{n-3} \cdots\left(b_{q}+2\right)_{n-3}} \frac{m^{n-3}}{(n-3) !}\right. \\
& \left.+2 \frac{a_{1} \cdots a_{p}}{b_{1} \cdots b_{q}} \sum_{n=2}^{\infty} \frac{\left(a_{1}+1\right)_{n-2} \cdots\left(a_{p}+1\right)_{n-2}}{\left(b_{1}+1\right)_{n-2} \cdots\left(b_{q}+1\right)_{n-2}} \frac{m^{n-2}}{(n-2) !}\right] \\
= & \frac{1}{{ }_{p} F_{q}(m)}\left[\frac { a _ { 1 } ( a _ { 1 } + 1 ) \cdots a _ { p } ( a _ { p } + 1 ) } { b _ { 1 } ( b _ { 1 } + 1 ) \cdots b _ { q } ( b _ { q } + 1 ) } m ^ { 2 } { } _ { p } F _ { q } \left(a_{1}\right.\right. \\
& \left.\left.+2 ; b_{1}+2 ; m\right)+2 \frac{a_{1} \cdots a_{p}}{b_{1} \cdots b_{q}} m_{p} F_{q}\left(a_{1}+1 ; b_{1}+1 ; m\right)\right] \\
\leq & \frac{1}{\beta+2}
\end{aligned}
$$

by the given hypothesis. This completes the proof of Theorem 2.3.

Theorem 2.4 Let $a_{i}, b_{j}>0(i=1,2, \ldots, p ; j=1,2, \ldots, q)$, and let $f \in \mathscr{R}^{\tau}(A, B)$. Suppose that the inequality

$$
\begin{aligned}
& \frac{(A-B)|\tau|}{{ }_{p} F_{q}(m)}\left[(1+\beta) \frac{a_{1} \cdots a_{p}}{b_{1} \cdots b_{q}} m_{p} F_{q}\left(a_{1}+1 ; b_{1}+1 ; m\right)+(1-\alpha)\left({ }_{p} F_{q}(m)-1\right)\right] \\
& \quad \leq 1-\alpha
\end{aligned}
$$


holds with one of the following conditions:

(1) $p \leq q$ and $m>0$,

(2) $p=q+1$ and $m<1$,

(3) $p=q+1, m=1$, and $\sum_{j=1}^{q} b_{j}>\sum_{i=1}^{p} a_{i}+1$.

Then $\Omega(p, q, m) f \in \mathscr{U} \mathscr{C} \mathscr{V}(\alpha, \beta)$.

Proof Since

$$
\Omega(p, q, m) f(z)=z+\sum_{n=2}^{\infty} \frac{\left(a_{1}\right)_{n-1} \cdots\left(a_{p}\right)_{n-1}}{\left(b_{1}\right)_{n-1} \cdots\left(b_{q}\right)_{n-1}} \frac{m^{n-1}}{(n-1) !} \frac{a_{n} z^{n}}{F_{q}(m)},
$$

to prove that $\Omega(p, q, m) f \in \mathscr{U} \mathscr{C} \mathscr{V}(\alpha, \beta)$, by Lemma 2.2 it suffices to prove that

$$
\sum_{n=2}^{\infty} n[n(1+\beta)-(\alpha+\beta)] \frac{\left(a_{1}\right)_{n-1} \cdots\left(a_{p}\right)_{n-1}}{\left(b_{1}\right)_{n-1} \cdots\left(b_{q}\right)_{n-1}} \frac{m^{n-1}}{(n-1) !} \frac{\left|a_{n}\right|}{{ }_{p} F_{q}(m)} \leq 1-\alpha .
$$

Using Lemma 2.4, we have

$$
\begin{aligned}
& \sum_{n=2}^{\infty} n[n(1+\beta)-(\alpha+\beta)] \frac{\left(a_{1}\right)_{n-1} \cdots\left(a_{p}\right)_{n-1}}{\left(b_{1}\right)_{n-1} \cdots\left(b_{q}\right)_{n-1}} \frac{m^{n-1}}{(n-1) !} \frac{\left|a_{n}\right|}{{ }_{p} F_{q}(m)} \\
& \leq \frac{(A-B)|\tau|}{{ }_{p} F_{q}(m)} \sum_{n=2}^{\infty}[n(1+\beta)-(\alpha+\beta)] \frac{\left(a_{1}\right)_{n-1} \cdots\left(a_{p}\right)_{n-1}}{\left(b_{1}\right)_{n-1} \cdots\left(b_{q}\right)_{n-1}} \frac{m^{n-1}}{(n-1) !} \\
&= \frac{(A-B)|\tau|}{{ }_{p} F_{q}(m)}\left[(1+\beta) \sum_{n=2}^{\infty}(n-1) \frac{\left(a_{1}\right)_{n-1} \cdots\left(a_{p}\right)_{n-1}}{\left(b_{1}\right)_{n-1} \cdots\left(b_{q}\right)_{n-1}} \frac{m^{n-1}}{(n-1) !}\right. \\
&\left.+(1-\alpha) \sum_{n=2}^{\infty} \frac{\left(a_{1}\right)_{n-1} \cdots\left(a_{p}\right)_{n-1}}{\left(b_{1}\right)_{n-1} \cdots\left(b_{q}\right)_{n-1}} \frac{m^{n-1}}{(n-1) !}\right] \\
&=\left.\frac{(A-B)|\tau|}{{ }_{p} F_{q}(m)}\left[(1+\beta) \frac{a_{1} \cdots a_{p}}{b_{1} \cdots b_{q}} m_{p} F_{q}\left(a_{1}+1, b_{1}+1, m\right)+(1-\alpha){ }_{p} F_{q}(m)-1\right)\right] \\
& \leq 1-\alpha
\end{aligned}
$$

by the given hypothesis. This completes the proof of Theorem 2.4.

Theorem 2.5 Let $a_{i}, b_{j}>0(i=1,2, \ldots, p ; j=1,2, \ldots, q)$, and let $f \in \mathscr{R}^{\tau}(A, B)$. Suppose that the inequality

$$
\frac{(A-B)|\tau|}{{ }_{p} F_{q}(m)}\left[\frac{a_{1} \cdots a_{p}}{b_{1} \cdots b_{q}} m_{p} F_{q}\left(a_{1}+1 ; b_{1}+1 ; m\right)\right] \leq \frac{1}{\beta+2}
$$

holds with one of the following conditions:

(1) $p \leq q$ and $m>0$,

(2) $p=q+1$ and $m<1$,

(3) $p=q+1, m=1$, and $\sum_{j=1}^{q} b_{j}>\sum_{i=1}^{p} a_{i}+1$.

Then $\Omega(p, q, m) f \in \mathscr{U} \mathscr{C} \mathscr{V}(\beta)$.

Proof The proof of Theorem 2.5 is similar to that of Theorem 2.4, and therefore we omit it. 
Remark 2.3 If we put $p=q=1$ in Theorems 2.1 and 2.2, then we obtain the corresponding results of Porwal [14].

\section{An integral operator}

In this section, we obtain analogous results in connection with the particular integral

$$
G(p, q, m, z)=\int_{0}^{z} \frac{{ }_{p} F_{q}(m, t)}{t} d t .
$$

Theorem 3.1 If all the conditions of Theorem 2.2 hold, then $G(p, q, m, z)$ defined by (3.1) is in the class $\mathscr{U} \mathscr{C} \mathscr{V}(\alpha, \beta)$.

Proof From representation (3.1) we have

$$
G(p, q, m, z)=z+\sum_{n=2}^{\infty} \frac{\left(a_{1}\right)_{n-1} \cdots\left(a_{p}\right)_{n-1}}{\left(b_{1}\right)_{n-1} \cdots\left(b_{q}\right)_{n-1}} \frac{m^{n-1}}{n !} \frac{z^{n}}{{ }_{p} F_{q}(m)} .
$$

To prove that $G(p, q, m, z) \in \mathscr{U} \mathscr{C} \mathscr{V}(\alpha, \beta)$, by Lemma 2.2 it suffices to prove that

$$
\sum_{n=2}^{\infty} n[n(1+\beta)-(\alpha+\beta)] \frac{\left(a_{1}\right)_{n-1} \cdots\left(a_{p}\right)_{n-1}}{\left(b_{1}\right)_{n-1} \cdots\left(b_{q}\right)_{n-1}} \frac{m^{n-1}}{n !} \frac{1}{{ }_{p} F_{q}(m)} \leq 1-\alpha .
$$

We have

$$
\begin{aligned}
\sum_{n=2}^{\infty} n[n(1+\beta)-(\alpha+\beta)] \frac{\left(a_{1}\right)_{n-1} \cdots\left(a_{p}\right)_{n-1}}{\left(b_{1}\right)_{n-1} \cdots\left(b_{q}\right)_{n-1}} \frac{m^{n-1}}{n !} \frac{1}{{ }_{p} F_{q}(m)} \\
=\frac{1}{{ }_{p} F_{q}(m)} \sum_{n=2}^{\infty}[n(1+\beta)-(\alpha+\beta)] \frac{\left(a_{1}\right)_{n-1} \cdots\left(a_{p}\right)_{n-1}}{\left(b_{1}\right)_{n-1} \cdots\left(b_{q}\right)_{n-1}} \frac{m^{n-1}}{(n-1) !} \\
=\frac{1}{{ }_{p} F_{q}(m)} \sum_{n=2}^{\infty}[(1+\beta)(n-1)+1-\alpha] \frac{\left(a_{1}\right)_{n-1} \cdots\left(a_{p}\right)_{n-1}}{\left(b_{1}\right)_{n-1} \cdots\left(b_{q}\right)_{n-1}} \frac{m^{n-1}}{(n-1) !} \\
=\frac{1}{{ }_{p} F_{q}(m)}\left[(1+\beta) \sum_{n=2}^{\infty} \frac{\left(a_{1}\right)_{n-1} \cdots\left(a_{p}\right)_{n-1}}{\left(b_{1}\right)_{n-1} \cdots\left(b_{q}\right)_{n-1}} \frac{m^{n-1}}{(n-2) !}\right. \\
\left.\quad+(1-\alpha) \sum_{n=2}^{\infty} \frac{\left(a_{1}\right)_{n-1} \cdots\left(a_{p}\right)_{n-1}}{\left(b_{1}\right)_{n-1} \cdots\left(b_{q}\right)_{n-1}} \frac{m^{n-1}}{(n-1) !}\right] \\
=\frac{1}{{ }_{p} F_{q}(m)}\left[(1+\beta) \frac{a_{1} \cdots a_{p}}{b_{1} \cdots b_{q}} m_{p} F_{q}\left(a_{1}+1 ; b_{1}+1 ; m\right)+(1-\alpha)\left({ }_{p} F_{q}(m)-1\right)\right] .
\end{aligned}
$$

The last expression is bounded above by $1-\alpha$ if (2.6) holds. Thus the proof of Theorem 3.1 is established.

Theorem 3.2 Let $a_{i}, b_{j}>0(i=1,2, \ldots, p ; j=1,2, \ldots, q)$. Suppose that the inequality

$$
\frac{a_{1} \cdots a_{p}}{b_{1} \cdots b_{q}} m_{p} F_{q}\left(a_{1}+1 ; b_{1}+1 ; m\right) \leq \frac{{ }_{p} F_{q}(m)}{\beta+2}
$$

holds with one of the following conditions: 
(1) $p \leq q$ and $m>0$,

(2) $p=q+1$ and $m<1$,

(3) $p=q+1, m=1$, and $\sum_{j=1}^{q} b_{j}>\sum_{i=1}^{p} a_{i}+1$.

Then $G(p, q, m, z)$ defined by (3.1) is in the class $\mathscr{U} \mathscr{C} \mathscr{V}(\beta)$.

Proof The proof of this theorem is much akin to that of Theorem 3.1. Therefore we omit it.

Remark 3.1 If we put $p=2$ and $q=1$ in Theorems $2.1-3.2$, then we obtain the corresponding results of Porwal and Gupta [15].

\section{Acknowledgements}

The authors are thankful to the referee for valuable comments and observations, which helped in improving the paper. We also wish to express our sincere appreciation to Prof. K.K. Dixit for his valuable suggestions and encouragements.

\section{Funding}

Not applicable.

\section{Availability of data and materials}

Not applicable.

\section{Competing interests}

The authors declare that they have no competing interests.

\section{Authors' contributions}

All authors equally worked on the results, and they read and approved the final manuscript.

\section{Author details}

1 Post-Graduate and Research Department of Mathematics, Voorhees College, Vellore 632 001, Tamilnadu, India. ${ }^{2}$ Department of Mathematics, Ram Sahai Government Degree College, Bairi-Shivrajpur, Kanpur 209205, Uttar Pradesh, India. ${ }^{3}$ Post-Graduate and Research Department of Mathematics, Govt Arts College (Men), Krishnagiri 635001 , Tamilnadu, India.

\section{Publisher's Note}

Springer Nature remains neutral with regard to jurisdictional claims in published maps and institutional affiliations.

Received: 18 March 2020 Accepted: 13 November 2020 Published online: 23 November 2020

\section{References}

1. Ahmad, M.S., Mehmood, Q., Nazeer, W., Haq, A.U.: An application of a hypergeometric distribution series on certain analytic functions. Sci. Int. 27, 2989-2992 (2015)

2. Bajpai, D.: A study of univalent functions associated with distribution series and $q$-calculus. M.Phil. Dissertation, CSJM University, Kanpur, India (2016)

3. Bharati, R., Parvatham, R., Swaminathan, A.: On subclasses of uniformly convex functions and corresponding class of starlike functions. Tamkang J. Math. 28(1), 17-32 (1997)

4. Dixit, K.K., Pal, S.K.: On a class of univalent functions related to complex order. Indian J. Pure Appl. Math. 26(9), 889-896 (1995)

5. El-Deeb, S.M., Bulboacă, T., Dziok, J.: Pascal distribution series connected with certain subclasses of univalent functions. Kyungpook Math. J. 59(2), 301-314 (2019)

6. Goodman, A.W.: On uniformly convex functions. Ann. Pol. Math. 56(1), 87-92 (1991)

7. Goodman, A.W.: On uniformly starlike functions. J. Math. Anal. Appl. 155(2), 364-370 (1991)

8. Kanas, S., Wisniowska, A.: Conic regions and k-uniform convexity. J. Comput. Appl. Math. 105(1-2), 327-336 (1999)

9. Kanas, S., Wiśniowska, A.: Conic domains and starlike functions. Rev. Roum. Math. Pures Appl. 45(4), 647-657 (2000)

10. Ma, W.C., Minda, D.: Uniformly convex functions. Ann. Pol. Math. 57(2), 165-175 (1992)

11. Nazeer, W., Mehmood, Q., Kang, S.M., Haq, A.U.: An application of binomial distribution series on certain analytic functions. J. Comput. Anal. Appl. 26, 11-17 (2019)

12. Porwal, S.: An application of a Poisson distribution series on certain analytic functions. J. Complex Anal. 2014, Article ID 984135 (2014)

13. Porwal, S.: Generalized distribution and its geometric properties associated with univalent functions. J. Complex Anal. 2018, Article ID 8654506 (2018)

14. Porwal, S.: Confluent hypergeometric distribution and its applications on certain classes of univalent functions of conic regions. Kyungpook Math. J. 58(3), 495-505 (2018)

15. Porwal, S., Gupta, A.: Some properties of convolution for hypergeometric distribution type series on certain analytic univalent functions. Acta Univ. Apulensis, Mat.-Inform. 56, 69-80 (2018)

16. Rainville, E.D.: Special Functions. Macmillan Co., New York (1960)

17. Robertson, M.S.: On the theory of univalent functions. Ann. Math. 37(2), 374-408 (1936)

18. Silverman, H.: Univalent functions with negative coefficients. Proc. Am. Math. Soc. 51, 109-116 (1975) 